\title{
SELEÇÃO DE MATERIAIS PARA FABRICAÇÃO DE COMPONENTES AERONÁUTICOS EM COMPÓSITOS INTEGRANDO ASPECTOS AMBIENTAIS E ECONÔMICOS *
}

\author{
Celise Villa dos Santos ${ }^{1}$ \\ Bráulio Salumão de Oliveira² \\ Daniel Rodrigo Leiva ${ }^{3}$ \\ Leandro Innocentini Lopes de Faria ${ }^{4}$ \\ José Ângelo Rodrigues Gregolin ${ }^{5}$
}

\section{Resumo}

Este estudo avaliou oportunidades de integração entre requisitos ambientais e econômicos durante etapas iniciais do desenvolvimento de produtos. Como principal ferramenta foi utilizado o Mapeamento de Fluxo de Valor, associando conceitos de manufatura enxuta, Design for Manufacturing and Assembly (DFMA) e Ecodesign, aplicado a um item de mobiliário aeronáutico em material compósito. Os resultados apontam que o tempo total de fabricação poderia ser reduzido em até $43 \%$, além da otimização logística. A forma de construção influencia diretamente os impactos ambientais e oportunidades de utilização de matérias-primas menos agressivas foram apontadas como sugestões. O aspecto visual dos Mapas de Fluxo de Valor facilita a análise das informações e a tomada de decisão na seleção de materiais e processos que minimizem desperdícios e impactos ambientas na manufatura.

Palavras-chave: Seleção de Materiais; Compósitos; Sustentabilidade; Aeronaves.

\section{MATERIALS SELECTION FOR MANUFACTURING AERONAUTICAL COMPONENTS IN COMPOSITES INTEGRATING ENVIRONMENTAL AND ECONOMIC ISSUES}

\section{Abstract}

This study evaluated opportunities for integration between environmental and economic requirements during the early stages of product development. The main analytical tool used was Value Stream Mapping, associating concepts of lean manufacturing, Design for Manufacturing and Assembly (DFMA) and Ecodesign. They were applied to a case study involving an aircraft interior cabinet. The results indicate that total manufacturing time could be $43 \%$ lower, besides logistic optimization. The cabinet construction influences the environmental impacts, and opportunities for using less aggressive raw materials were suggested. The visual approach of Value Stream Maps facilitates information analysis and decision making for materials and processes selection that minimizes waste and environmental impacts in manufacturing.

Keywords: Materials Selection; Composites; Sustainability; Aircrafts.

1 Engenheira de Materiais, Doutora em Ciência e Engenharia de Materiais, Pesquisadora e PósDoutoranda, Núcleo de Informação Tecnológica em Materiais, UFSCar, São Carlos-SP, Brasil.

2 Engenheiro de Materiais, Mestre em Ciência e Engenharia de Materiais, Doutorando, Programa de Pós-graduação em Ciência e Engenharia de Materiais, UFSCar, São Carlos-SP, Brasil.

3 Engenheiro de Materiais, Doutor em Ciência e Engenharia de Materiais, Professor, Departamento de Engenharia de Materiais, UFSCar, São Carlos-SP, Brasil.

4 Engenheiro de Materiais, Doutor em Ciência e Engenharia de Materiais, Professor, Departamento de Ciência da Informação, UFSCar, São Carlos-SP, Brasil.

5 Engenheiro de Materiais, Doutor em Engenharia Mecânica, Professor Sênior, Departamento de Engenharia de Materiais, UFSCar, São Carlos-SP, Brasil. 


\section{INTRODUÇÃO}

Os materiais compósitos têm sido estratégicos na produção de componentes aeronáuticos por propiciarem redução de peso aliada a desempenho mecânico, tanto em estruturas externas como internas de aeronaves. Exemplos de aplicação em estruturas internas são painéis de revestimento de teto e laterais, bagageiros, portas, divisórias e gabinetes [1,2].

Estes materiais também têm sido considerados promissores na redução de impactos ambientais na fabricação das aeronaves e para a produção de componentes de modo simplificado e com menores custos [3]. Ambos desafios tecnológicos vão ao encontro da atual tendência de requisitos de sustentabilidade ambiental e econômica para bens de consumo, e afetam a seleção de materiais e processos no desenvolvimento de produtos [4].

Análises de impacto ambiental, considerando todas as etapas do ciclo de vida das aeronaves, indicam que o consumo de combustíveis durante o voo é responsável por cerca de $90 \%$ do impacto total. O uso de materiais de baixo peso, como os compósitos, teria papel fundamental para redução deste impacto, embora apresentem restrições ambientais relacionadas, por exemplo, a dificuldades de descarte e reciclagem no final do ciclo de vida do produto. Estas restrições, embora minimizadas pelo baixo volume de aeronaves produzidas em comparação com outros bens de consumo, e o longo tempo de vida útil do produto, tem sido alvo de pesquisas e patentes na indústria aeronáutica $[2,5,6]$.

A produção de estruturas de materiais compósitos com custo reduzido vem sendo relacionada ao desenvolvimento de produtos e à utilização de processos de fabricação automatizados. No desenvolvimento de produtos, por exemplo, busca-se a otimização dos materiais e processos para evitar custos recorrentes e, na automação, procura-se empregar técnicas como como RTM (Resin Transfer Molding), RFI (Resin Film Infusion), Fiber-placement, entre outras. [3]. Entretanto, a automação não necessariamente se justifica para a produção de itens em pequeno volume e muitas vezes personalizada, como estruturas do interior de aeronaves para uso executivo [7].

No desenvolvimento do produto, limitações para a otimização econômica e ambiental da manufatura de componentes em material compósito do interior de aeronaves podem ser relacionadas à concepção baseada em poucos materiais de uso já consolidado, e selecionados por critérios fortemente influenciados pelo design do produto [8]. Essas limitações também podem ser relacionadas às dificuldades para a articulação entre projeto - seleção de materiais - seleção de processos, ambiental e economicamente sustentáveis, como também tem sido reportado no desenvolvimento de produtos de um modo geral [9, 10]. A baixa articulação desde os estágios iniciais do desenvolvimento do produto pode resultar, em geral, na necessidade de aplicação de medidas de ajustes nos estágios pós-desenvolvimento, desde amanufatura até final do ciclo de vida, o que muitas vezes está limitado pelas decisões anteriores sem a articulação inicial[4, 11].

As principais metodologias aplicadas no desenvolvimento dos produtos para consideração de aspectos ambientais em geral se baseiam em Estudos de Avaliação do Ciclo de Vida do Produto, em guias de Projeto para o Ambiente (Ecodesign) e em ferramentas como as denominadas Ashby Eco-audits tools. A partir de estudos de Avaliação do Ciclo de Vida, são definidas prioridades para minimização do impacto ambiental dos produtos relativas aos materiais e processos. A realização desses estudos, entretanto, requer informações, não necessariamente existentes, sobre 0 
desempenho dos materiais e processos a serem utilizados. Os guias de Projeto para - Ambiente trazem orientações gerais para a seleção de materiais e processos definidos a partir de estudos de análise do ciclo de vida. Dada a carência de informações específicas sobre os produtos, ferramentas como a Ashby Eco Adit Tools priorizam a análise de impactos como consumo de água, energia, e emissões de dióxido de carbono [4, 11-16].

A articulação entre projeto - processos é abordada na literatura principalmente pela metodologia Projeto para Fabricação e Montagem (DFMA - Design for Manufacturing and Assembly). Ela faz uso de conhecimentos existentes, baseados na experiência, de fatores de que auxiliam ou prejudicam a produtividade na fabricação de um produto. Entretanto, apresenta triangulação limitada em relação à seleção dos materiais por indicar recomendações genéricas, como reduzir o número de materiais no componente, ou utilizar materiais disponíveis e bem conhecidos para minimizar incertezas. Adicionalmente, tende a avaliar os processos que compõem a fabricação do produto de maneira individualizada, desconsiderando combinações que possibilitem, por exemplo: - fluxos de produção contínuos; - baixos volumes de estoques intermediários; - flexibilidade para alternar fabricação de produtos personalizados, entre outros [17, 18].

$O$ atendimento ao conjunto das novas necessidades e desafios para que a fabricação de aeronaves tenha sustentabilidade ambiental e econômica requer uma reflexão, e eventual mudança, nos métodos de concepção e desenvolvimento dos produtos. Para isso, é importante combinar métodos que possam potencializar resultados para o fortalecimento do produto e de sua manufatura, promovendo uma visão integrada das informações essenciais para a tomada de decisão [10, 19].

Uma metodologia que pode auxiliar nesta integração é a de Mapeamento de Fluxo de Valor, que se baseia nos conceitos de manufatura enxuta e que tem sido empregada para análise da integração da operação da manufatura com questões ambientais inerentes, em diferentes tipos de produtos e setores industriais, como na produção de aeronaves, automóveis, e de laminados de madeira para acabamento de móveis [20, 21, 22, 23].

O Mapeamento de Fluxo de Valor, desenvolvido e consolidado na indústria automobilística para melhoria de processos em operação, disseminou-se na indústria aeronáutica entre os principais fabricantes de aeronaves, tanto para otimização da manufatura, como dos processos administrativos, e no desenvolvimento dos produtos [24, 25]. No desenvolvimento de produtos, as aplicações principais se referem à melhoria da utilização de recursos de engenharia e redução do tempo entre a concepção e o lançamento [26, 27]. Entretanto, verificase uma carência de publicações que associem os conceitos de manufatura enxuta com a seleção de materiais e processos.

O objetivo do presente trabalho é avaliar a aplicação da metodologia Mapeamento de Fluxo de Valor, associando conceitos de manufatura enxuta e de sustentabilidade ambiental, para identificar e priorizar possíveis requisitos de projeto do produto, materiais e processos, que visam fortalecer a integração entre estes elementos nas etapas iniciais do desenvolvimento dos produtos.

\section{MATERIAIS E MÉTODOS}

A metodologia de Mapeamento de Fluxo de Valor foi empregada na fase de produção de um componente do mobiliário do interior de aeronaves, como principal ferramenta analítica para investigar e priorizar requisitos de projeto, materiais e 
processos que, aplicados nas etapas iniciais do desenvolvimento dos produtos, possam resultar em benefícios econômicos e ambientais.

Com o propósito de obter uma visão integrada entre as questões de manufatura e ambientais, foram realizadas as seguintes etapas e atividades:

Etapa 1: Definição de um produto para estudo de caso, que representasse uma amostra do conjunto dos componentes em material compósito do interior de aeronaves, em termos de materiais empregados, técnicas de construção e processos de fabricação.

Etapa 2: Mapeamento do Fluxo de Valor atual para o produto do estudo de caso conforme procedimentos e notação de Rother e Shook [20]. Neste mapeamento, procurou-se identificar oportunidades de otimização no conjunto das etapas do processo de fabricação em atividades que não agregam valor ao produto na perspectiva dos clientes.

As atividades que não agregam valor foram classificadas em: - Necessárias, devido ao nível tecnológico atual, não podendo ser removidas a não ser que os materiais e processos sejam modificados, e - Desperdícios, como defeitos, excesso de produção, estoques, processamento desnecessário, transporte desnecessário e espera por fatores como falta de estoque ou gargalos no processamento.

Etapa 3: Mapeamento do Fluxo de Valor ambiental para o produto do estudo de caso, com adaptação da representação das informações do modelo proposto por Rother e Shook [20]. A adaptação abrangeu registros de categorias de indicadores de impactos ambientais na manufatura nas dimensões ambiental, social e econômica indicadas no quadro 1 [11, 15], e baseou-se em trabalhos de Torres e Gatti (2009) [28] e Moraes et al (2014) [29].

A definição do produto a focalizar e o desenho dos Mapas de Fluxo de Valor foram realizados mediante levantamento de campo em linhas de produção de móveis para interiores de aeronaves, entrevistas com especialistas, feiras e catálogos de aeronaves e de materiais.

O produto do estudo de caso é o gabinete modelo hipotético mostrado na Figura 1. As dimensões escolhidas para o gabinete são mais representativas da tecnologia de fabricação, do que para seu tamanho e da complexidade, e correspondem a $60 \times 40$ x $45 \mathrm{~cm}$ (largura x profundidade x altura).

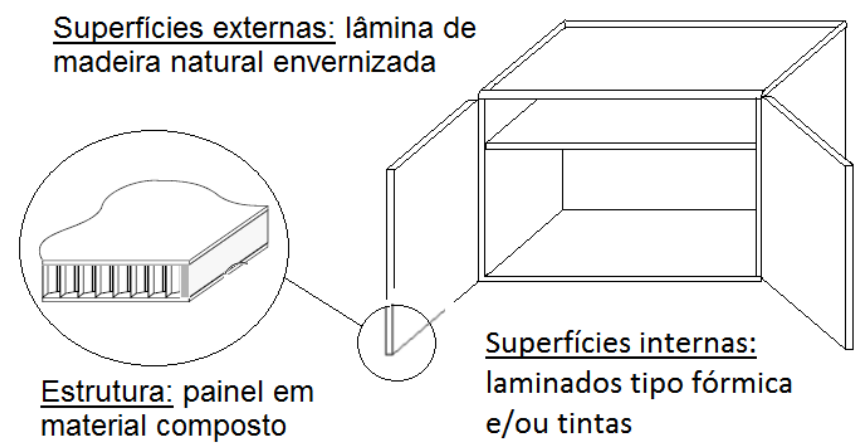

Figura 1. llustração do gabinete modelo utilizado como estudo de caso

O gabinete é constituído $90 \%$ em volume de placas de material compósito com faces de resina fenólica ou epóxi reforçadas com fibra de vidro, e núcleo de poliaramida em forma de colmeia [30]. As bordas destas placas são preenchidas com resinas epóxi para possibilitar aplicação de materiais de acabamento. A junção dos componentes é realizada com elementos metálicos como pinos, insertos e 
dobradiças, fixados com adesivos do tipo epóxi. Os materiais de acabamento podem ser:

- Laminados sintéticos de alta pressão (tipo fórmica), ou de madeira natural, colados normalmente com adesivo de contato à base de neoprene;

- Vernizes poliuretânicos, acrílicos ou poliéster, de base solvente e cura por polimerização a baixas temperaturas, aplicados sobre a madeira;

- Tintas de base solvente ou água, sistema poliuretânico, e cura por polimerização a baixas temperaturas, aplicadas diretamente sobre as placas de material compósito.

Quadro 1. Categorias de indicadores e forma de análise dos impactos ambientais na fabricação de um componente para interior de aeronaves

\begin{tabular}{|c|c|c|c|}
\hline $\begin{array}{l}\text { Dimensão da } \\
\text { sustentabilidade }\end{array}$ & $\begin{array}{l}\text { Categoria de } \\
\text { indicador }\end{array}$ & Grupo & Análise dos impactos \\
\hline \multirow{5}{*}{ Ambiental } & \multirow[b]{2}{*}{ Impacto ambiental } & Químico & $\begin{array}{l}\text { Uso de materiais tóxicos. } \\
\text { Geração de resíduos perigosos. } \\
\text { Poluição da água, ar ou solo. }\end{array}$ \\
\hline & & Físico & $\begin{array}{l}\text { Uso de materiais que degradam ambientes naturais } \\
\text { Quantidade resíduos de materiais gerados no } \\
\text { processamento. } \\
\text { Reuso ou reciclagem dos resíduos. } \\
\text { Disposição do produto no final do ciclo de vida. }\end{array}$ \\
\hline & \multirow{3}{*}{$\begin{array}{l}\text { Consumo de } \\
\text { recursos }\end{array}$} & Energia & $\begin{array}{l}\text { Tipo e quantidade de energia consumida para: } \\
\text { Processamento das matérias-primas. } \\
\text { Contenção e disposição de resíduos. } \\
\text { Transporte de matérias-primas e do produto final } \\
\text { até o ponto de uso. }\end{array}$ \\
\hline & & Solo & $\begin{array}{l}\text { Impacto dos materiais e processos na necessidade } \\
\text { de infraestrutura para fabricação do produto. } \\
\text { Uso do solo para disposição de resíduos. }\end{array}$ \\
\hline & & Materiais & $\begin{array}{l}\text { Eficiência no uso dos materiais: } \\
\% \text { de matérias-primas convertidas em produto. } \\
\text { Perdas de material de suporte do processo produtivo } \\
\text { (material de consumo) ou resíduos de fabricação. } \\
\text { Volume, tipo e destino de embalagens de matérias- } \\
\text { primas e do produto acabado. }\end{array}$ \\
\hline \multirow{4}{*}{ Social } & Consumidor & \multicolumn{2}{|c|}{ Riscos à saúde e segurança dos consumidores. } \\
\hline & Sociedade & \multirow{2}{*}{\multicolumn{2}{|c|}{$\begin{array}{l}\text { Oportunidades de envolvimento das comunidades locais. } \\
\text { Riscos físicos: lesões por esforços repetitivos. }\end{array}$}} \\
\hline & \multirow{2}{*}{ Trabalhadores } & & \\
\hline & & \multicolumn{2}{|c|}{ Riscos químicos: doenças por exposição a produtos químicos. } \\
\hline \multirow[b]{2}{*}{ Econômica } & Capital humano & \multicolumn{2}{|c|}{ Gastos com saúde e segurança dos trabalhadores. } \\
\hline & Custos financeiros & \multicolumn{2}{|c|}{ Gastos com proteção ambiental. } \\
\hline
\end{tabular}

As técnicas de construção e fabricação, e o agrupamento dos processos, são similares aos encontrados na indústria de mobiliário geral (residências, escritórios, hotéis) para a fabricação de móveis artesanais e de baixo volume de produção. São técnicas que envolvem investimento relativamente baixo em máquinas e equipamentos, principalmente em centros de usinagem de baixa precisão e complexidade para corte de placas de painel sanduíche. As dimensões da estrutura do móvel normalmente são ajustadas durante a montagem das peças sem acabamento, e por isso não necessitam de precisão dimensional. O acabamento é aplicado nas estruturas montadas.

Etapa 4: Análise dos mapas de fluxo de valor, identificando de modo integrado as principais características de projeto, materiais e processos, para melhor 
desempenho econômico e ambiental no desenvolvimento de novos produtos. As potencialidades foram comparadas, avaliando e recomendando possíveis soluções. Considerou-se a redução do tempo total de fabricação o principal objetivo de otimização para desempenho econômico, visto que é um importante elemento de custo. A redução dos tempos possibilita maior flexibilidade e agilidade no fornecimento dos produtos, redução do ciclo financeiro, redução dos estoques e das áreas ocupadas para fabricação e armazenagem [20]. As oportunidades identificadas foram comparadas com as orientações presentes em guias de DFMA $[7,17,31]$. Na análise dos impactos ambientais, as oportunidades identificadas conforme critérios do Quadro 1 foram comparados com orientações para Ecodesing gerais e específicas para a indústria aeronáutica [11, 15, 32].

\section{RESULTADOS E DISCUSSÃO}

\subsection{Mapeamento de fluxo de valor - Análise de perdas e do impacto ambiental dos materiais e processos de fabricação}

O mapeamento do fluxo de valor e a análise dos tempos de agregação de valor de cada uma das etapas de fabricação do gabinete modelo, mostrados nas Figuras 2 e 3 , indicam que somente $11 \%$ das atividades agregam valor ao produto, $79 \%$ são necessárias apenas em função da tecnologia de processamento adotada para os materiais utilizados, e 10\% podem ser consideradas desperdício puro.

As atividades necessárias, impostas pelas tecnologias de materiais e processos, estão relacionadas principalmente a Etapa 5, de envernizamento e pintura. Esta etapa consome 9 dias ou 44\% do ciclo de produção do gabinete. Nestes processos, $78 \%$ do seu tempo (149 h) são consumidos em atividades de cura das resinas.

Os desperdícios puros que correspondem a $10 \%$ do tempo total de fabricação do gabinete, e que poderiam ser eliminados com ajustes e melhorias de processo, estão relacionadas principalmente à Etapa 3 de Pré-Montagem. Esta etapa só é necessária porque a precisão de corte das peças na Etapa 1 é baixa. A precisão de usinagem das peças depende das propriedades do material compósito e do equipamento utilizado. Quanto maior a precisão do equipamento, maior o seu custo. A Etapa de pré-montagem poderia ser eliminada ao utilizar, por exemplo, soluções da indústria moveleira tradicional [33]. Entretanto requereria, além de investimentos em equipamentos de corte mais precisos, alterações no projeto aeronáutico que priorizasse o design com superfícies planas, e com técnicas de junção adequadas e esteticamente viáveis para as peças já com acabamento.

A eliminação da Etapa de Pré-Montagem, além de reduzir o tempo total de fabricação do móvel, também poderia trazer ganhos logísticos em termos de volume transportado, visto que o móvel poderia ser levado desmontado até o local de instalação na aeronave, minimizando também impactos ambientais relativos ao transporte e embalagem. A finalização da montagem do móvel no local de instalação (Etapa 6 da Figura 3) reduziria o volume de transporte do gabinete modelo, por exemplo, em aproximadamente $50 \%$. Isto poderia ser uma vantagem quando as distâncias entre o local de produção do mobiliário e o de sua instalação na aeronave transpõem países e continentes, o que é bastante frequente. 


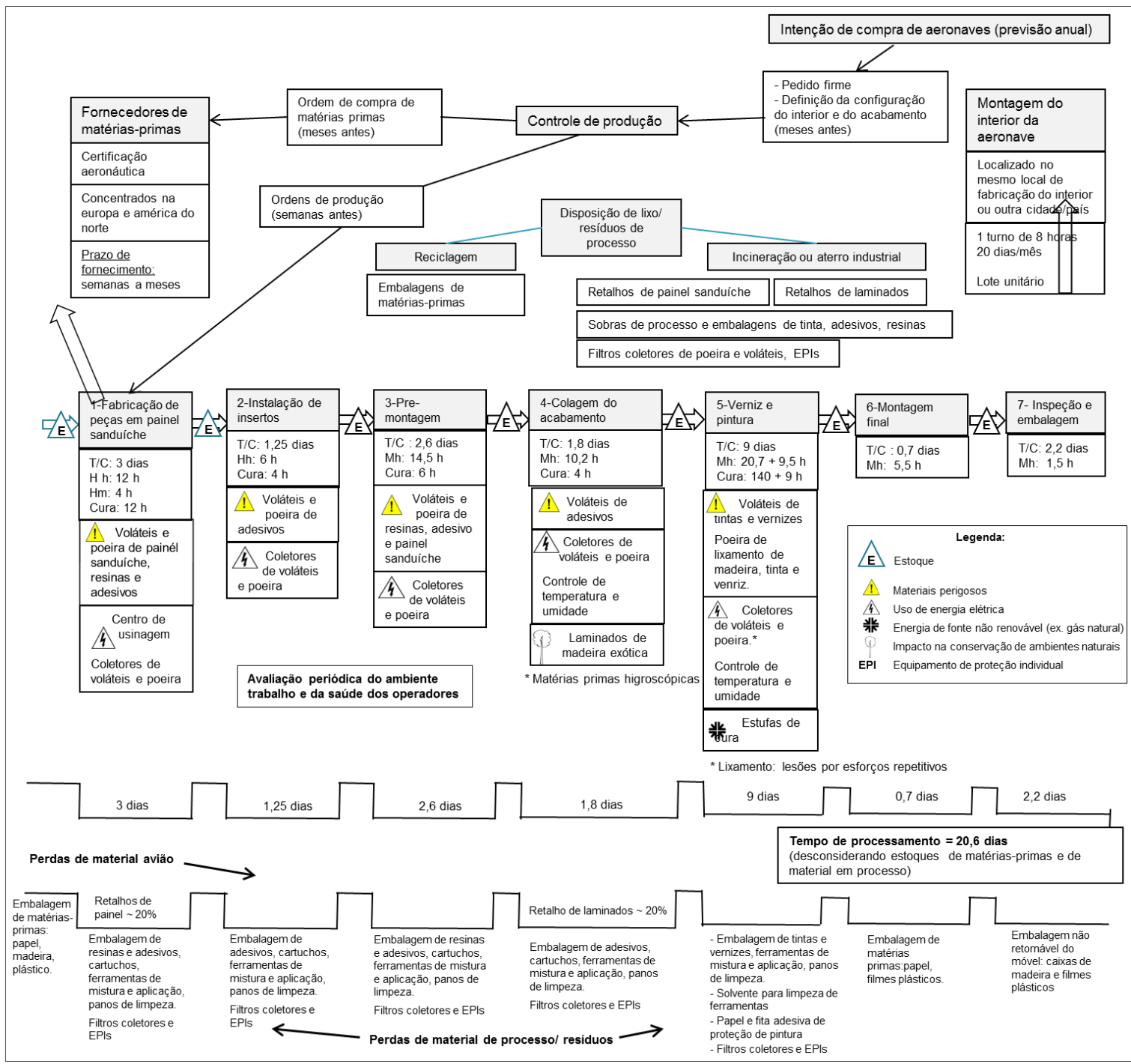

Figura 2. Mapeamento de fluxo de valor com considerações ambientais para o gabinete modelo

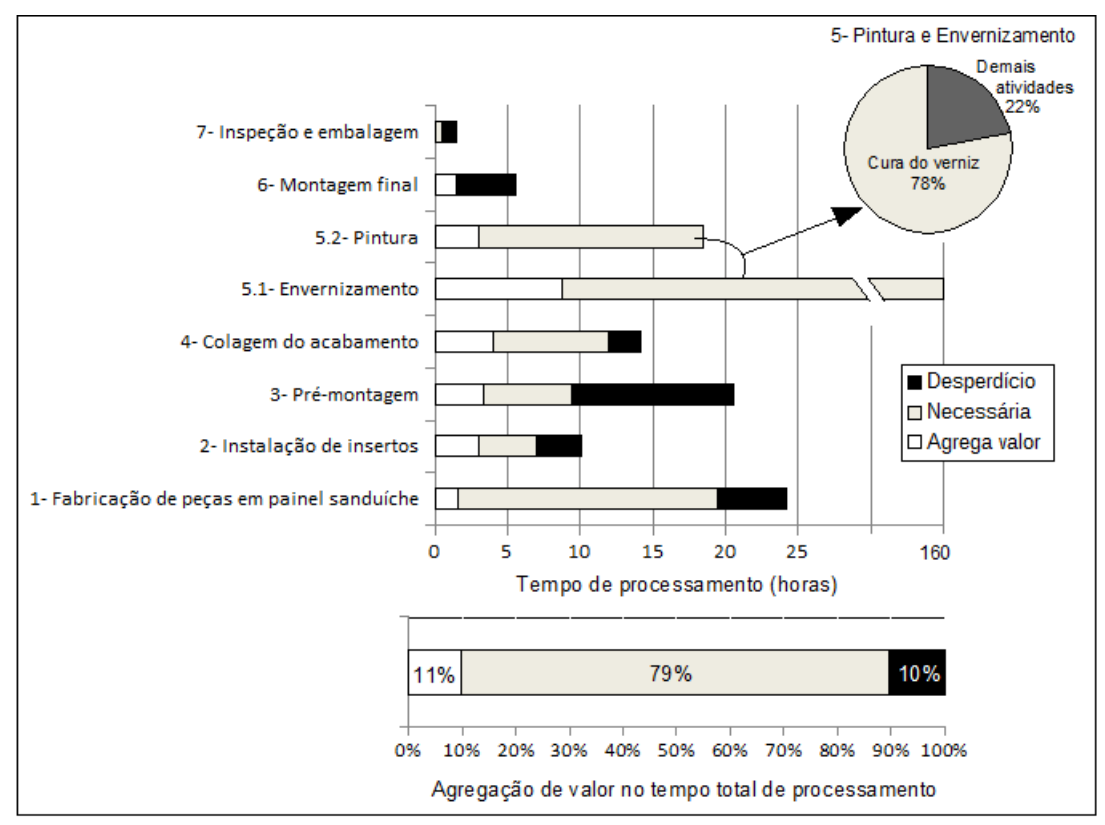

Figura 3. Distribuição dos tempos de processamento entre as etapas de fabricação do gabinete modelo, e agregação de valor em cada etapa. 
A expectativa de redução do tempo total de fabricação do gabinete pela utilização de vernizes de cura rápida e eliminação da atividade de pré-montagem poderia ser de até $43 \%$. Estima-se possibilidade de redução de 9 para 2,8 dias no processo de aplicação de verniz e tintas se utilizada, por exemplo, a tecnologia de cura por radiação ultravioleta. Esta tecnologia, consolidada na indústria moveleira tradicional, utiliza formulações à base de resinas acriladas e tem como vantagens ambientais, em relação às formulações que utilizam cura por calor evaporação de solventes, economia energética e o baixo teor de solventes e voláteis. Os principais desafios para a uso desta tecnologia em móveis aeronáuticos está no desenvolvimento de formulações com aditivos antichamas, e de técnicas de junção de material compósito que possibilitem a montagem dos móveis após aplicação dos vernizes ou tintas. Isso porque esta tecnologia de cura requer a aproximação uniforme da lâmpada de cura em todas as superfícies das peças e a aplicação prioritariamente em sistemas fechados tipo túnel com esteira [34].

As informações ambientais coletadas no mapeamento indicam que os impactos são relativos principalmente às propriedades dos atuais materiais utilizados e ao conceito e processos de fabricação dos móveis. Em quase todas as atividades de fabricação são liberados voláteis orgânicos durante o manuseio e cura de adesivos, resinas, tintas e vernizes. $O$ uso destes materiais requer materiais de consumo não recicláveis como recipientes plásticos, panos com solventes de limpeza, que também necessitam de descarte especial, por incineração ou em aterros industriais para materiais perigosos [35]. Poeiras são geradas em operações de corte, furação e lixamento de painéis sanduíche, de resinas, e de materiais de acabamento como laminados, tintas e vernizes.

Os voláteis orgânicos e as poeiras são prejudiciais à saúde dos trabalhadores e necessitam de monitoramento periódico. Além do monitoramento, são necessários sistemas industriais de coleta e contenção destes resíduos, em conjunto com sistemas de controle de temperatura e umidade requeridos para a preservação físico-química dos materiais de acabamento e seus adesivos, que são, em geral, higroscópicos [36, 31, 36, 37]. Esses sistemas são grandes consumidores de energia não associada diretamente aos processos de construção do gabinete modelo.

O uso de laminados de madeira como material de acabamento (Etapa 4 da Figura 3) tem impacto na conservação dos ambientes naturais quando são utilizadas madeiras exóticas, principalmente nas aeronaves de grande porte e de maior custo.

A forma de construção do gabinete também influencia diretamente os impactos ambientais da manufatura. A realização da Etapa 3, de Pré-Montagem, com a fixação da estrutura do gabinete antes da aplicação do acabamento requer maior área de produção, maiores bancadas de trabalho, cabines de aplicação e estufas para cura de tintas e vernizes. O trabalho em áreas maiores também requer maior consumo energético para controle de temperatura, umidade, e sistemas de exaustão. Outro impacto é na ergonomia, pela dificuldade de acesso nas operações de aplicação de materiais de acabamento, como na colagem de laminados e na aplicação de tintas e vernizes. Adicionalmente, a fixação da estrutura do móvel antes da aplicação do acabamento dificulta a automação de atividades como lixamento, em que a coleta e contenção de poeira poderiam ser facilitadas, além da possibilidade de eliminar potencial causa de lesões por esforço repetitivo.

A realização de Pré-montagem, quando faz uso de insertos metálicos ao invés de resinas, também traz impactos ambientais pelo aumento da dificuldade de 
disposição do gabinete no final do ciclo de vida do produto, por fazer uso de um material de origem diferente dos painéis estruturais.

Os atuais fornecedores de matérias-primas para interiores de aeronaves estão concentrados na América do Norte e Europa, onde se localizam as duas maiores indústrias aeronáuticas, Boeing e Airbus. Os custos logísticos e de volume de estoques, embora não levantados quantitativamente neste trabalho, podem ser significativos para os fabricantes de interiores aeronáuticos localizados fora deste eixo, como é o caso do Brasil. Nestes casos, o desenvolvimento de fornecedores locais poderia ser considerado para redução, inclusive, do impacto ambiental do produto.

\subsection{Melhorias para a redução de perdas e do impacto ambiental}

As possíveis melhorias para redução de perdas e do impacto ambiental para o gabinete do estudo de caso indicam orientações específicas que poderiam ser utilizadas nas etapas indiciais do desenvolvimento de novos produtos para a seleção dos materiais e processos.

As orientações são relativas principalmente à: - substituição de vernizes e tintas por composições de baixo teor de solventes e de cura rápida; - ao uso de materiais de acabamento e adesivos mais estáveis quando sujeitos a variações de temperatura e umidade, e, - e ao uso de matérias-primas de fontes renováveis com reduzida emissão de voláteis e poeiras no processamento. Em relação ao conceito de fabricação dos móveis, mudanças nos processos de fabricação e de junção estrutural das peças em material compósito para melhor precisão dimensional e possibilidade de eliminação da pré-montagem dos componentes.

Todas as orientações identificadas puderam ser diretamente relacionadas com as orientações gerais apresentadas nos guias de DFMA e de Ecodesign, mostradas nos Quadros 2 e 3, indicando o potencial da metodologia aplicada para extrair e sintetizar informações da experiência e prática industrial de um produto específico.

Quadro 2. Orientações para redução de perdas no gabinete modelo e comparação com critérios baseados em DFMA

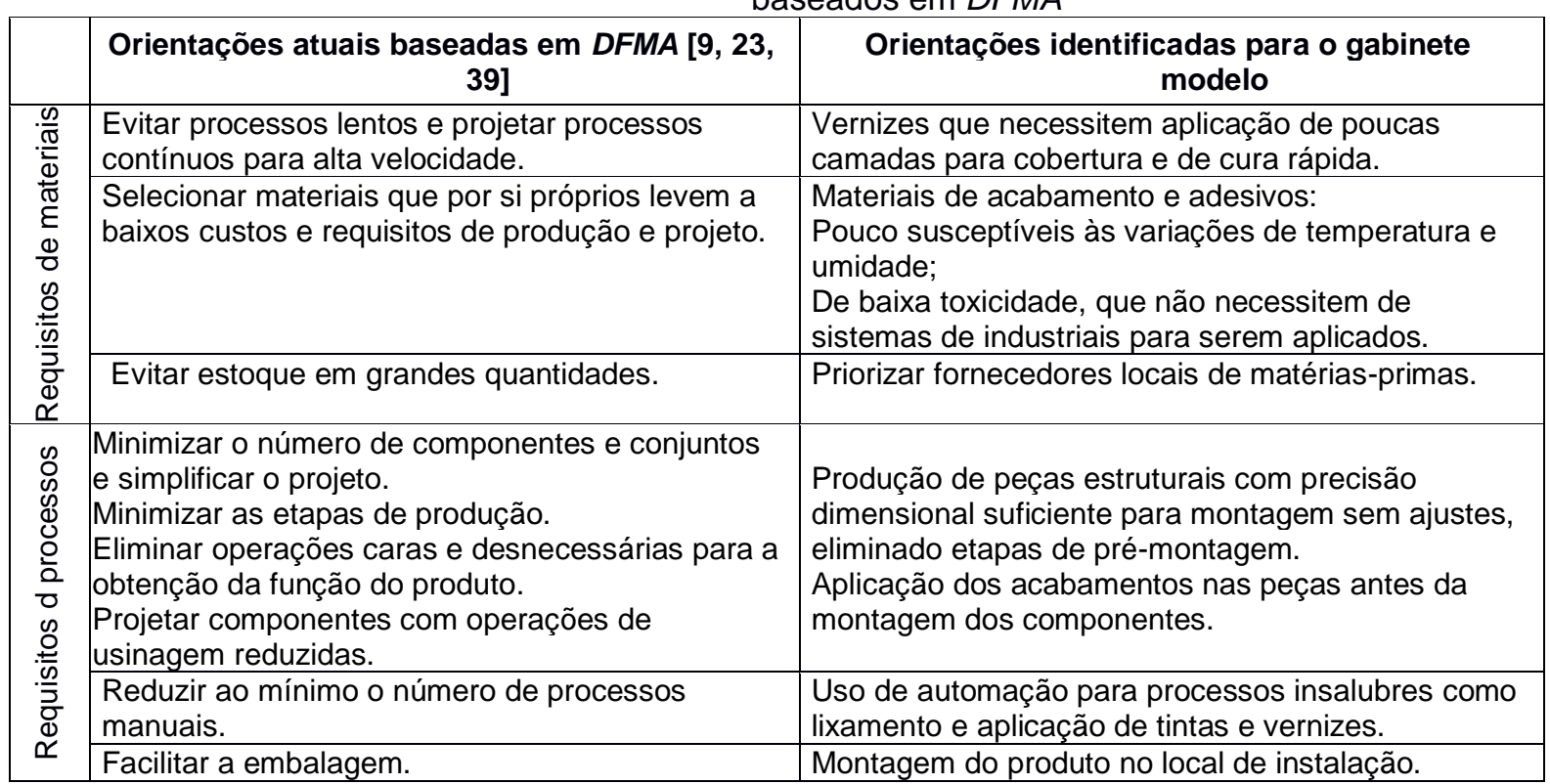


Quadro 3. Orientações para melhoria da sustentabilidade ambiental do gabinete modelo e comparação com critérios atualmente utilizados nos projetos aeronáuticos

\begin{tabular}{|c|c|c|}
\hline & $\begin{array}{l}\text { Requisitos atuais para sustentabilidade } \\
\text { do projeto aeronáutico }[13,20]\end{array}$ & $\begin{array}{c}\text { Orientações para melhoria da sustentabilidade do } \\
\text { gabinete modelo }\end{array}$ \\
\hline \multirow{3}{*}{ 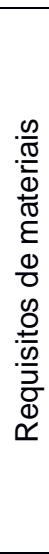 } & $\begin{array}{l}\text { Ausência de substâncias tóxicas aos seres } \\
\text { humanos e ao ambiente. }\end{array}$ & $\begin{array}{l}\text { Redução de voláteis orgânicos nos materiais de } \\
\text { acabamento e adesivos. }\end{array}$ \\
\hline & $\begin{array}{l}\text { Uso eficiente dos recursos: } \\
\text { Disponibilidade dos materiais. } \\
\text { Origem sustentável dos materiais. } \\
\text { Quantidade e tipo de energia e recursos. } \\
\text { para extração e produção primária das } \\
\text { matérias-primas. }\end{array}$ & $\begin{array}{l}\text { Evitar uso de laminados de madeira exótica. } \\
\text { Alternativas para vernizes com alto consumo energético } \\
\text { para cura. } \\
\text { Baixa susceptibilidade dos materiais às condições } \\
\text { ambientais para evitar consumo energético no } \\
\text { armazenamento e processamento. } \\
\text { Fornecedores locais de matérias primas para reduzir } \\
\text { consumo de recursos para transporte. }\end{array}$ \\
\hline & $\begin{array}{l}\text { Quantidade reduzida e boa qualidade dos } \\
\text { resíduos gerados na produção das matérias- } \\
\text { primas e na manufatura. } \\
\text { Opções sustentáveis para disposição dos } \\
\text { componentes no final do ciclo de vida. } \\
\end{array}$ & $\begin{array}{l}\text { Técnicas de fixação que evitem insertos metálicos } \\
\text { (material de origem diferente do estrutural). } \\
\text { Alternativas sustentáveis para os atuais painéis } \\
\text { estruturais, e uso de materiais de acabamento de } \\
\text { natureza compatível. }\end{array}$ \\
\hline \multirow[b]{2}{*}{ 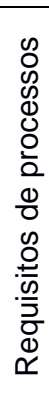 } & $\begin{array}{l}\text { Baixa toxidade química e física dos materiais } \\
\text { aeronáuticos e de consumo do processo } \\
\text { produtivo. }\end{array}$ & $\begin{array}{l}\text { Automação de operações insalubres (lixamento e } \\
\text { aplicação de tintas/vernizes) } \\
\text { Montagem sem ajustes para reduzir o uso de resinas }\end{array}$ \\
\hline & $\begin{array}{l}\text { Eficiência no uso de recursos: } \\
\text { Quantidade e tipo de energia. } \\
\text { Quantidade de materiais aeronáuticos e de } \\
\text { consumo. } \\
\text { Minimização da poluição: } \\
\text { Tipo e quantidade de descartes atmosféricos } \\
\text { e na água } \\
\text { Tipo e quantidade de resíduos sólidos }\end{array}$ & $\begin{array}{l}\text { Redução de perdas de matérias-primas na produção de } \\
\text { painéis e no corte de laminados de acabamento. } \\
\text { Transporte dos móveis desmontados até o local de } \\
\text { instalação na aeronave. }\end{array}$ \\
\hline
\end{tabular}

A estratégia de uso da metodologia Mapeamento de Fluxo de Valor aplicada no estudo também permitiu a visualização integrada dos aspectos ambientais e econômicos para a identificação e priorização de requisitos de materiais e processos que talvez não pudessem ser facilmente identificados se analisados separadamente.

As potenciais soluções identificadas apresentam sinergia entre si para melhoria tanto dos aspectos ambientais como econômicos. Este efeito sinérgico tem sido indicado por diversos autores [21, 22, 38], inclusive em relatório da Agência de Proteção Ambiental Norte Americana [23].

\section{CONCLUSÃo}

A metodologia de Mapeamento de fluxo de valor mostrou-se adequada para integrar questões ambientais e de otimização da manufatura na articulação de requisitos de materiais e de processos de fabricação a serem utilizados como parâmetros no desenvolvimento de novos produtos de interiores de aeronaves.

A metodologia proposta possibilita 0 desdobramento e a especificação de orientações para a otimização da manufatura e melhoria ambiental propostas pelos guias de DFMA e Ecodesign, combinando e sintetizando informações da experiência e prática industrial.

A abordagem visual dos mapas de fluxo de valor possibilita a compilação de informações essenciais e a combinação com informações de outras fontes para a tomada de decisão, o que pode facilita a análise e o consenso entre técnicos e decisores de diferentes áreas e formações. Os mapas também podem ser 
adaptados em função dos indicadores de produção e de sustentabilidade mais adequados para cada produto.

O mapeamento em linhas de produção reais e voltadas para o desenvolvimento de produtos específicos pode enriquecer a análise, trazendo outros elementos para priorização de planos de mudança. Informações sobre o custo e volume de estoques reais, por exemplo, podem ser utilizadas para identificar materiais críticos que devem ser substituídos, e que pode implicar na necessidade de desenvolvimento de novos fornecedores ou novos insumos.

\section{Agradecimentos}

Os autores agradecem à CAPES, ao CNPq, ao PPGCEM/UFSCar, ao PPGCTS/UFSCar e ao NIT/Materiais UFSCar pelo apoio prestado.

\section{REFERÊNCIAS}

1 Black SARA. Advanced materials for aircraft interiors. High performance composites. 2006; 14 (6): 24.

2 Heitzmann M, Lindenberger B, Ng Ching Tai; Airbus Operations Ltd. Composite material. WO2010142774 (A1). 2010.

3 Agência Brasileira de Desenvolvimento Industrial. Estudo prospectivo aeronáutico: relatório geral. Centro de Gestão e Estudos Estratégicos. 2009; 14.

4 Ashby M. Green design in a resource constrained world - a materials perspective. Granta Webseminar, 2015.

5 Howe S, Kolios AJ, Brennan FP. Environmental life cycle assessment of commercial passenger jet airliners. Transportation Research Part D. 2013; 19: 34-41.

6 Scelsi L et al. Potential emissions savings of lightweight composite aircraft components evaluated through life cycle assessment. Express Polymer Letters. 2011;5(3):209-217.

7 Barbosa GF, Carvalho J, Filho, EGV. A proper framework for design of aircraft production system based on lean manufacturing principles focusing to automated processes. The International Journal of Advanced Manufacturing Technology. 2014;72(9-12):1257-1273.

8 Costa, FR. Situação atual e perspectivas de utilização de materiais no interior de aeronaves executivas [Dissertação]. São Carlos: Univ. Federal de São Carlos; 2009.

9 Albiñana JC, Vila C. A framework for concurrent material and process selection during conceptual product design stages. Materials \& Design. 2012; 41:433-446.

10 Ilgin, MA, Gupta SM. Environmentally conscious manufacturing and product recovery (ECMPRO): a review of the state of the art. Journal of Environmental Management. 2010; 91 (3): 563-591.

11 AIRBUS - ACADEMY. Design for environment. 2008 [Acesso em: jun. 2017]. Disponível em: ec.europa.eu/environment/life/project/Projects/.

12 Ashby MF. Materials and the environment: eco-informed material choice. United Kingdom: Elsevier; 2012.

13 Eddy DC et al. A predictive modelling-based material selection method for sustainable product design. Journal of Engineering Design. 2015; 26 (10-12):365-390.

14 Zarandi $\mathrm{MH}$. F et al. A material selection methodology and expert system for sustainable product design. The International Journal of Advanced Manufacturing Technology. 2011; $57(9-12): 885-903$

15 Pigosso DCA. Ecodesign maturity model: a framework to support companies in the selection and implementation of ecodesign practices [Tese]. São Carlos: Escola de Engenharia de São Carlos; 2012.

16 Dallara E, Kusnitz J, Bradley M. Parametric life cycle assessment for the design of aircraft. SAE Int. J. Aerosp. 2013 (2): 736-745.

17 Edwards K. Towards more strategic product design for manufacture and assembly: priorities for concurrent engineering. Materials and Design. 2002; 23: 651-656. 
18 Landru DA, Bréchet YA, Salvo LB. Materials and process selection - an integrated approach. Advanced Engineering Materials. 2002; 4(6): 357-362.

19 Buchert T et al. Enabling product development engineers to select and combine methods for sustainable design. Procedia CIRP. 2014; 15: 413-418.

20 Rother M, Shook J. Aprendendo a enxergar. São Paulo: Lean Institute Brasil; 2003.

21 Santos A. Vezzoli C, Morais Junior D. Linking lean production and design for sustainability on the issue of waste reduction. Product: Management \& Development. 2008; 6(2):135-141.

22 Pampanelli AB, Found P, Bernardes AM. A lean \& green model for a production cell. Journal of cleaner production. 2014; 85: 19-30.

23 United Environmental Protection Agency (EPA). Lean manufacturing and the environment: research on advanced manufacturing systems and the environment and recommendations for leveraging better environmental performance. 2003 [Acesso em: jun. 2017]. Disponível em: epa.gov/lean.

24 Airbus. Lean. 2014 [Acesso em jun. 2017]. Disponível em: airbus.com/work/why-joinairbus/training-and-development/lean/.

25 Jenkins M. Boeing Frontiers. Getting lean. 2002 [Acesso em: jun. 2017]; 1(4). Disponível em: boeing.com/news/frontiers/archive/2002/august/cover.html.

26 Al-Ashaab A et al. The transformation of product development process into lean environment using set-based concurrent engineering: a case study from an aerospace industry. Concurrent Engineering Research and Applications. 2013;21 (4): 268-285.

27 Smith S. Applying lean techniques to the engineering process. SAE Int. J. Aerosp.2009; $1(1): 757-769$.

28 Torres, A.S., \& Gati, A.M. (2009). Environmental Value Stream Mapping (EVSM) as sustainability management tool. Picmet: Portland International Conference on Management of Engineering \& Technology; 2009; Portland. 2009. p. 1689-1698.

29 Moraes AJl et al. Proposta de integração dos indicadores de sustentabilidade no mapeamento do fluxo de valor em um sistema de produção. XXXIV Encontro nacional de engenharia de produção; 2014; Curitiba.

30 Hexcel. Sandwich panel fabrication technology. 2001 [Acesso em: jun. 2017]. Disponível em: hexcel.com/Resources/Technology-Manuals.

31 Herrera A. Design for manufacture and assembly, makes a difference among the boeing lean design initiatives. SAE Technical Paper.1998; 981873.

32 Doudrich G. et al. Defining Environmental indicators at detail design stage as part of an ecodesign strategy. SAE Int. J. Aerosp. 2013 (2):727-735.

33 Sebrae/ESPM. Móveis para cozinha - estudos de mercado. 2008 [Acesso em: jun. 2017]. Disponível em: //bis.sebrae.com.br/.

34 Kelly M. The Economics of Sustainable UV Technology. Radtech Report. 2009 [Acesso em: jul. 2017]. Disponível em:

72.52.184.8/ radtecho/pdfs/EconomicsSustainableUVTechnology.pdf.

35 Huntsman. Aerospace materials for production and assembly. 2013 [Acesso em: jun. 2017]. Disponível em: http://www.huntsman.com/advanced materials/a/Home.

36 Nascimento GC. Avaliação da influência de intempéries na qualidade superficial de sistemas poliméricos utilizados em acabamento de mobiliário aeronáutico [Tese]. São José dos Campos: Instituto Tecnológico de Aeronáutica; 2013.

37 Wilsonart. Wilsonart® cabinet liner sheets. 2016 [Acesso em: jun. 2017]. Disponível em: wilsonart.com/resources.

38 Simões CL. et al. Integrating environmental and economic life cycle analysis in product development: a material selection case study. The International Journal of Life Cycle Assessment. 2013; 18 (9):1734-1746. 